\title{
PHASE SPACE AS ARENA FOR ATOMIC MOTION IN A QUANTIZED LIGHT FIELD
}

\author{
E. Mayr, D. Krähmer, A.M. IIerkommer, V.M. Akulin*, W.P. Schleich ${ }^{\dagger}$ \\ Abtcilung für Quantenphysik, Universität Ulm, 89069 Ulm, Germany
}

AND I.SII. AVERBUKIII

Department of Chemical Physics, Weizmann Institute of Science, Rehovot 76100, Israel

We describe the motion of an atom in a quantized light field using the concept of the Wigner distribution in phase space. This approach provides a pictorial explanation of the deflection and focusing of the atomic beam.

PACS numbers: 42.50.Vk, 42.50.Wm

\section{Introduction}

In classical mechanics a phase space distribution contains the complete information about a dynamical system. This concept carrics over to the quantum world: a prominent distribution is the Wigner function [1]. This representation of quantum mechanics gives immediate insight into the dynamics of a quantum system. In the present paper we analyse the mechanical action of a quantized light field [2] on a non-resonant atom using this concept [3].

The paper is organized as follows. In Sec. 2 we present the model [4] describing the motion of a non-resonant atom in a single mode of a quantized light field. Starting from the Schrödinger equation for the state vector for the atomic motion and the field we derive the equation of motion for the Wigner function of the atom. The latter turns out to be the sum of the Wigner functions corresponding to the motion of the atom in the individual number states, weighted with the photon statistics. In Sec. 3 we give an analytical solution for the equation of motion of the Wigner function for the case when the wavelength of the light is much larger than the de Broglie wavelength of the atom [5]. Making use of this small parameter we expand the actual light potential in a Taylor series up to second order and obtain a first order differential equation solvable exactly. We devote Sec. 4 to a discussion of the distribution of atoms after the interaction with the light field. Following the evolution of the Wigner function we note that each individual Fock state deflects

*Also at Moscow Instilule of Physics and Technology, Dolgoprudny, Moscovskaja Obl., Russian Federation.

IAlso at Max-Planck-Institut für Quantenoptik, 85748 Garching bei München, Germany. 
the atoms in different directions [6-8] and focuses [9] them at different points. In Sec. 5 we derive simple expressions for the position and the size of the foci of the individual Fock states as it follows from the phase space dynamics. We conclude by summarizing our main results in Sec. 6 .

\section{Formulation of the problem}

We describe the interaction of a non-resonant atom and a quantized electromagnetic field mode shown in Fig. 1 by the effective Hamiltonian [4, 10-12]

$$
\hat{H}=\frac{\hat{p}_{x}^{2}}{2 M}+[\theta(z+L)-\theta(z)] g(x) \hat{a}^{\dagger} \hat{a} .
$$

The operators $\hat{a}$ and $\hat{a}^{\dagger}$ are the annihilation and creation operators of the ficld mode and the coupling constant $g(x)=\alpha \mathcal{E}_{0}^{2}(x)$ is the atomic linear susceptibility $\alpha$ multiplied by the "square of the electric field per photon". For simplicity we assume a rectangular field distribution in $z$-direction of length $L$ as expressed by the IIeaviside step functions $\theta(z)$. Because of the non-resonant interaction we neglect the internal degrees of freedom of the atom. The $z$-direction we call longitudinal and the $x$-direction transverse. IIere we treat the transverse motion of the atom quantum mechanically and hence keep the operator nature of the kinetic energy $\hat{p}_{x}^{2} / 2 M$. In $z$-direction we considror the velocities such that the corresponding de Broglie wavelength $\lambda_{\mathrm{dB}}=2 \pi \hbar / M v_{z}$ is much smaller than the typical dimension of the electromagnetic field estimated by the wavelength $\lambda$. IIence we describe the motion along the $z$-axis classically. We therefore associate the $z$-coordinate with time via the relation $z=v_{z} t-L$ and consider the spatial evolution of the systcm in this direction as a time evolution given by the Schrödinger equation

$$
\mathrm{i} h \frac{\partial|\Psi\rangle}{\partial t}=\hat{H}|\Psi\rangle \text {. }
$$

For a given $L$ the velocity $v_{z}$ defines an interaction time $t_{L} \equiv L / v_{z}$.

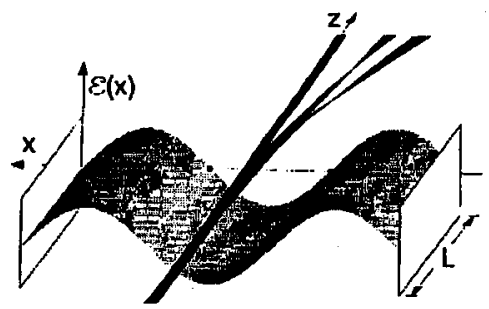

Fig. 1. Quantum lens. A beam of non-resonant atoms in $z$-direction interacts with the light ficld in the region $-L \leq z \leq 0$. Different Fock states deflect atoms in differcnt dircctions and focus them at different points. 
When the atom enters the field at $t=0$, the state vector $|\Psi(t=0)\rangle$ is the direct product of the transverse wave function $f(x)$ of the atom and the ficld state $|\psi\rangle$, that is

$$
|\Psi(t=0)\rangle=\sum_{n=0}^{\infty} w_{n}|n\rangle \otimes \int_{-\infty}^{\infty} \mathrm{d} x f(x)|x\rangle,
$$

where $w_{n}$ denote the photon probability amplitude of the field. The Schrödinger equation (2) with the IIamiltonian (1) couples the degrces of freedom of the field and the motion. As a result of the interaction the states of the field and the atom get strongly entangled. This allows us to gain information about one subsystem via the other. When we substitute the ansatz

$$
|\Psi(t)\rangle=\sum_{n=0}^{\infty} w_{n} \int_{-\infty}^{\infty} \mathrm{d} x \Psi_{n}(x, t)|x\rangle \otimes|n\rangle
$$

for the state vector $|\Psi\rangle$ into the Schrödinger equation (2) with the IIamiltonian (1) we find

$$
\mathrm{i} \hbar \frac{\partial \Psi_{n}(x, t)}{\partial t}=\left\{\frac{p_{x}^{2}}{2 M}+\left[\theta(t)-\theta\left(t-\iota_{L}\right)\right] g(x) n\right\} \Psi_{n}(x, t) .
$$

Each equation of this system is the Sclırödinger equation for a particle which moves in the potential

$$
V_{n}(x)=[O(z+L)-O(z)] g(x) n
$$

of the individual Fock states. It gives the probability amplitude $\Psi_{n}(x, t)$ of finding the atom at the time $t$ at the position $x$, and the field in the $n$-th Fock state.

For the time interval $0 \leq t \leq t_{L}$, that is when the atom is in the standing light field it feels the potential

$$
V_{n}(x)=g(x) n,
$$

whereas for $t_{L}<t$, when it is out of the cavity

$$
V_{n}(x)=0 .
$$

IIence the time evolution of the state $|\Psi\rangle$ of the combined system of the quantum field and the transverse motion is given by the time evolution of the probability amplitudes $\Psi_{n}(x, t)$ subject to the initial condition $\Psi_{n}(x, t=0)=f(x)$.

The state vector $|\Psi\rangle$ contains the complete information about the ficld and the atom and our further treatment depends on the specific question we want to address. We can for example consider the properties of the quantum field ignoring the transverse motion of the atom or vice versa concentrate on the motion while ignoring the field variables. Morcover we can, of course, investigate the entanglement between the atomic and field variables via joint measurements $[8,12]$. In the present paper, however, we confine ourselves to the measurements of the atomic motion only. We are interested in the distribution of atoms in phase space spanned by transverse position $x$ and momentum $p$. Therefore as the main tool we introduce the concept of the Wigner function. 
We start from the density matrix

$$
\begin{aligned}
\hat{\rho}= & |\Psi(t)\rangle\langle\Psi(t)|=\sum_{n^{\prime}, n^{\prime \prime}=0}^{\infty} w_{n^{\prime}} w_{n^{\prime \prime}}^{*} \\
& \times \int_{-\infty}^{\infty} \mathrm{d} x^{\prime} \int_{-\infty}^{\infty} \mathrm{d} x^{\prime \prime} \Psi_{n^{\prime}}\left(x^{\prime}, t\right) \Psi_{n^{\prime \prime}}^{*}\left(x^{\prime \prime}, t\right)\left|x^{\prime}\right\rangle\left\langle x^{\prime \prime}|\otimes| n^{\prime}\right\rangle\left\langle n^{\prime \prime}\right|
\end{aligned}
$$

of the entire system and trace over the field variables to find for the reduced atomic density matrix

$$
\hat{\rho}_{\mathrm{at}}=\sum_{n=0}^{\infty}\langle n|\hat{\rho}| n\rangle=\sum_{n=0}^{\infty}\left|w_{n}\right|^{2} \int_{-\infty}^{\infty} \mathrm{d} x^{\prime} \int_{-\infty}^{\infty} \mathrm{d} x^{\prime \prime} \Psi_{n}\left(x^{\prime}, t\right) \Psi_{n}^{*}\left(x^{\prime \prime}, t\right)\left|x^{\prime}\right\rangle\left\langle x^{\prime \prime}\right| .
$$

We obtain the Wigner function [1]

$$
P^{(\mathrm{W})}(x, p, t)=\frac{1}{2 \pi h} \int_{-\infty}^{\infty} \mathrm{d} \xi \mathrm{e}^{-\frac{1}{h} p \xi}\left\langle x+\frac{1}{2} \xi\left|\hat{\rho}_{\text {at }}\right| x-\frac{1}{2} \xi\right\rangle
$$

for the transverse atomic motion by substituting the atomic density operator Eq. (10) into Eq. (11) and we arrive at

$$
P^{(\mathrm{W})}(x, p, t)=\sum_{n=0}^{\infty}\left|w_{n}\right|^{2} P_{n}^{(\mathrm{W})}(x, p, t) .
$$

This is the incoherent supcrposition of the atomic Wigner functions

$$
P_{n}^{(\mathrm{W})}(x, p, t)=\frac{1}{2 \pi \hbar} \int_{-\infty}^{\infty} \mathrm{d} \xi \mathrm{e}^{-\frac{i}{\hbar} p \xi} \Psi_{n}^{*}\left(x-\frac{1}{2} \xi, t\right) \Psi_{n}\left(x+\frac{1}{2} \xi, t\right)
$$

each of which corresponds to the motion of the atom in the potential $V_{n}$ given by the $n$-th Fock state. The weight of the $n$-th Wigner function is the photon statistics $\left|w_{n}\right|^{2}$ of the initial field state.

The equation of motion for $P_{n}^{(W)}$ following from the equation of motion of the density operator reads [1]:

$$
\begin{aligned}
& \frac{\partial P_{n}^{(\mathrm{W})}(x, p, t)}{\partial t} \\
& =-\frac{p}{M} \frac{\partial P_{n}^{(\mathrm{W})}(x, p, l)}{\partial x}+\sum_{l=0}^{\infty} \frac{(\mathrm{i} i / 2)^{2 l}}{(2 l+1) !} \frac{\partial^{2 l+1} V_{n}(x)}{\partial x^{2 l+1}} \frac{\partial^{2 l+1} P_{n}^{(\mathrm{W})}(x, p, l)}{\partial p^{2 l+1}} .
\end{aligned}
$$

We conclude this section by noting that we can find the time dependence of the transverse atomic motion by solving either the Schrödinger equation (5) for the probability amplitude $\Psi_{n}$ or the equation of motion for the Wigner function Eq. (14). Since the latter involves the position as well as the momentum it is in general harder to solve than the Schrödinger equation, although for some special cases it is much more illustrative.

\section{Wigner function of atomic motion}

In the present section we consider the deflection and focusing of the atoms for a simple case which nevertheless displays very interesting plysical phenomena. We take the initial transverse wave packet to be narrow compared to the wavelength 
of the light field and the intcraction time $t_{L}$ short, so that the wave packet docs not change its position in $x$-direction considerably. In this case the equation of motion for the Wigner function can be solved exactly and immediately provides us via Eq. (12) the position and momentum distribution of the atoms.

Indeed, we take as the initial condition for $\Psi_{n}$ a Gaussian wave packet

$$
\Psi_{n}(x, t=0)=\int(x)=(\sqrt{\pi} d)^{-1 / 2} \exp \left[-\frac{1}{2}\left(\frac{x}{d}\right)^{2}\right],
$$

where $d$ denotes the width of the transverse distribution of atoms. In the case $d \ll \lambda$ we can expand the mode function $g(x)$, Eq. (1), around the centre $x=0$ of the wave packet, i.e.

$$
g(x)=g_{0}+g_{1} x+\frac{1}{2} g_{2} x^{2}+\ldots \approx g_{0}-\frac{1}{2} \frac{g_{1}^{2}}{g_{2}}+\frac{1}{2} g_{2}\left(x+\frac{g_{1}}{g_{2}}\right)^{2},
$$

wherc $g_{1}=\left.\frac{\mathrm{d} g}{\mathrm{~d} x}\right|_{x=0}$ and $g_{2}=\left.\frac{\mathrm{d}^{2} g}{\mathrm{~d} x^{2}}\right|_{x=0}$. By combining the linear and the quadratic contributions of the potential in a binomial we reduce the problem to the motion in the parabolic potential

$$
V_{n}(x)=V_{n}\left(x_{f}\right)+\frac{1}{2} M \omega_{n}^{2}\left(x-x_{f}\right)^{2}
$$

of the displaced harmonic oscillator with minimum $V_{n}\left(x_{f}\right)=\left(g_{0}-\frac{1}{2} g_{1}^{2} / g_{2}\right) n$ at $x_{f}=-g_{1} / g_{2}$ and frequency $\omega_{n}=\sqrt{n g_{2} / M}$. Note that the constant term $V_{n}\left(x_{f}\right)$ can be omitted from the Schrödinger equation

$$
\mathrm{i} \hbar \frac{\partial \Psi_{n}(x, t)}{\partial t}=\left[\frac{p_{x}^{2}}{2 M}+\frac{1}{2} M \omega_{n}^{2}\left(x-x_{f}\right)^{2}\right] \Psi_{n}(x, t)
$$

for the probability amplitude $\Psi_{n}$, since it only results in an irrelevant phase factor.

We can use the well-known Green function for the harmonic oscillator to solve the cquation for the time evolution of $\Psi_{n}$. Jlowever, in the present paper we pursue the more illustrative phase space approach using the Wigner function as discussed in Sec. 2. In the harmonic approximation only the term $l=0$ in the equation of motion (14) for $P_{n}^{(\mathrm{W})}$ contributes to the sum, since $\partial^{n} V / \partial x^{n}=0$ for all $n>2$, and we are left with the classical Liouville equation

$$
\frac{\partial P_{n}^{(\mathrm{W})}(x, p, t)}{\partial t}=-\frac{p}{M} \frac{\partial P_{n}^{(\mathrm{W})}(x, p, t)}{\partial x}+M \omega_{n}^{2}\left(x-x_{f}\right) \frac{\partial P_{n}^{(\mathrm{W})}(x, p, t)}{\partial p}
$$

for the Wigner function.

The approximation by a harmonic oscillator has a big advantage. Since the frequency $\omega_{n}$ of the harmonic oscillator is independent of the oscillator amplitude, all parts of the distribution move in phase space with the same angular velocity, and the Wigner function $P_{n}^{(W)}(x, p, t)$ at time $t$ follows by a rotation of the initial Wigner function $P_{n}^{(W)}(x, p, t=0)$ around the phase space point $\left(x_{f}, 0\right)$. Indeed, with the help of the method of characteristics [13] one obtains

$$
P_{n}^{(\mathrm{W})}\left(x, p, t \leq t_{L}\right)=P_{n}^{(\mathrm{W})}(\bar{x}(x, p, t), \bar{p}(x, p, t), t=0)
$$

with

$$
\bar{x}(x, p, l)=\left(x-x_{f}\right) \cos \left(\omega_{n} l\right)-\frac{p}{M \omega_{n}} \sin \left(\omega_{n} l\right)+x_{f},
$$




$$
\bar{p}(x, p, t)=p \cos \left(\omega_{n} t\right)+M \omega_{n}\left(x-x_{f}\right) \sin \left(\omega_{n} t\right),
$$

which satisfies Eq. (19). This Wigner function describes the distribution of atoms in phase space as long as they interact with the standing light wave, that is for $0 \leq t \leq t_{L}$. After the interaction, i.e. $t>t_{L}$, we have $V_{n}=0$. The free motion of the atoms corresponds to the time evolution of the Wigner function given by the equation

$$
\frac{\partial P_{n}^{(\mathrm{W})}(x, p, t)}{\partial t}=-\frac{p}{M} \frac{\partial P_{n}^{(\mathrm{W})}(x, p, t)}{\partial x}
$$

Again we can find the solution

$$
P_{n}^{(\mathrm{W})}\left(x, p, t>t_{L}\right)=P_{n}^{(\mathrm{W})}\left(x-\frac{p}{M}\left(t-t_{L}\right), p, t=t_{L}\right)
$$

with the initial condition $P_{n}^{(\mathrm{W})}\left(x, p, \iota=\iota_{L}\right)$ using the method of characteristics. From Eq. (23) we note that the particles move along trajectories of constant momentum, that is parallel to the $x$-axis.

As the initial condition $P_{n}^{(\mathrm{W})}(x, p, t=0)$ we take the Wigner function

$$
P^{(\mathrm{W})}(x, p, t=0)=\frac{1}{\pi h} \exp \left[-\left(\frac{x}{d}\right)^{2}-\left(\frac{d}{h} p\right)^{2}\right]
$$

corresponding to the initial transverse distribution $f(x)$, Eq. (15). Ilence the phase space distribution of particles inside the cavity reads

$$
\begin{aligned}
& P_{n}^{(\mathrm{W})}\left(x, p, t \leq t_{L}\right) \\
& \quad=\frac{1}{\pi \hbar} \exp \left[-\frac{1}{d^{2}}\left(\left(x-x_{f}\right) \cos \left(\omega_{n} l\right)-\frac{p}{M \omega_{n}} \sin \left(\omega_{n} l\right)+x_{f}\right)^{2}\right] \\
& \quad \times \exp \left[-\left(\frac{d}{\hbar}\right)^{2}\left(p \cos \left(\omega_{n} l\right)+\left(x-x_{f}\right) M \omega_{n} \sin \left(\omega_{n} t\right)\right)^{2}\right],
\end{aligned}
$$

where we have substituted the trajectories $\bar{x}$ and $\bar{p}$, Eq. (21), into the initial distribution Eq. (24).

Since we only observe the atoms after they have left the light field we now consider $t>t_{L}$. The distribution Eq. (25) for $t=t_{L}$ scrves as the initial condition for the Wigner function of frec motion evolving according to Eq. (22). With the help of Eq. (23) we arrive at

$$
\begin{aligned}
& P_{n}^{(\mathrm{W})}\left(x, p, t \geq t_{L}\right) \\
& =\frac{1}{\pi \hbar} \exp \left\{-\frac{1}{d^{2}}\left[\left(x-\frac{p}{M}\left(t-\iota_{L}\right)-x_{f}\right) \cos \varphi_{n}-\frac{p}{M \omega_{n}} \sin \varphi_{n}+x_{f}\right]^{2}\right\} \\
& \quad \times \exp \left\{-\left(\frac{d}{h}\right)^{2}\left[p \cos \varphi_{n}+\left(x-\frac{p}{M}\left(t-t_{L}\right)-x_{f}\right) M \omega_{n} \sin \varphi_{n}\right]^{2}\right\},
\end{aligned}
$$


where $\varphi_{n}=\omega_{n} t_{L}$. Moreover, we recall that time corresponds to the $z$-coordinate via $z=v_{z} t-L$ and this distribution reads

$$
\begin{aligned}
& P_{n}^{(\mathrm{W})}(x, p, z) \\
& \quad=\frac{1}{\pi \hbar} \exp \left\{-\frac{1}{d^{2}}\left[\left(x-\frac{p}{M v_{z}} z-x_{f}\right) \cos \varphi_{n}-\frac{p}{M \omega_{n}} \sin \varphi_{n}+x_{f}\right]^{2}\right\} \\
& \quad \times \exp \left\{-\left(\frac{d}{\hbar}\right)^{2}\left[p \cos \varphi_{n}+\left(x-\frac{p}{M v_{z}} z-x_{f}\right) M \omega_{n} \sin \varphi_{n}\right]^{2}\right\} .
\end{aligned}
$$

In Fig. 2 we show the contour lines of our initial Gaussian Wigner cigar (24) at the origin of phase space aligned along the $x$-axis. Duc to the motion of the

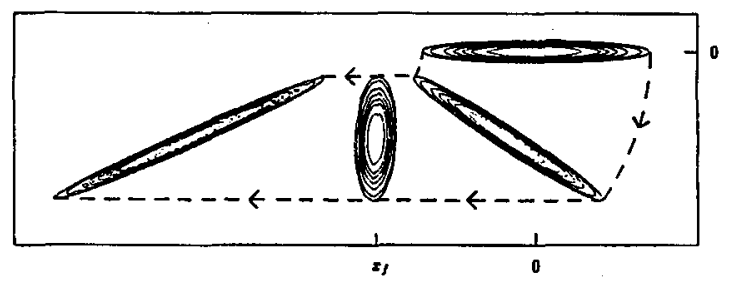

Fig. 2. Evolution of the atomic Wigner function in and after the light field for a Fock state $n=2$. In the light field the initial Gaussian cigar, narrow in momentum but broad in position, rotates following the evolution in the parabolic potential. Outside the light field the momentum is conserved which results in the shearing of the distribution. The width of the distribution in position reaches a minimum when the cigar stands vertically which corresponds to the focus.

atom in the harmonic oscillator potential $V_{n}$ the Gaussian cigar rotates in phase space around the point $\left(x=x_{f}, p=0\right)$ by the angle $\varphi_{n}=\omega_{n} t_{L}$, as given by Eq. (25). The following free evolution given by Eq. (27) is depicted in Fig. 2 for three typical times, that is for three typical positions outside of the light field, namely $z=0, z=z_{f}$ and $z=2 z_{f}$. We note that the width of the Wigner function in $x$-variable first decreases and then increases. It reaches a minimum when the cigar crosses the phase space line $x=x_{f}$. This is the physical origin of the focusing of the atoms.

So far we have only considered the motion of the atom in the potential $V_{n}$ given by the $n$-th Fock state. In the case of a field state $|\psi\rangle$ consisting of a superposition of Fock states the Wigner function $P^{(\mathrm{W})}(x, p, z)$ of the atomic motion is the incoherent sum Eq. (12) of the Wigner functions $P_{n}^{(\mathrm{W})}(x, p, t)$ weighted with the photon statistics $\left|w_{n}\right|^{2}$. In Fig. 3 we show the Wigner function $P^{(\mathrm{W})}(x, p, t)$ at the exit of the cavity, whereas in Fig. 4 we depict its contour lines for various times $t$, that is for various positions $z$. The left column displays the evolution of the initial Gaussian cigar in the standing light field. We note that duc to the $n$-dependence of the frequency $\omega_{n}$ of the oscillator potential, that is the angular velocity in phase space, the Gaussian cigar splits into many cigars - each of which 


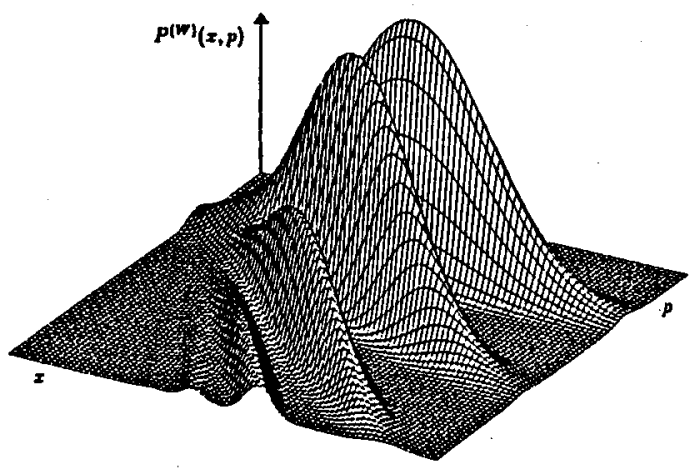

Fig. 3. Wigner function of the atom at the exit of the cavity. The field is in a colherent state of a verage number of photons $\bar{n}=1$.
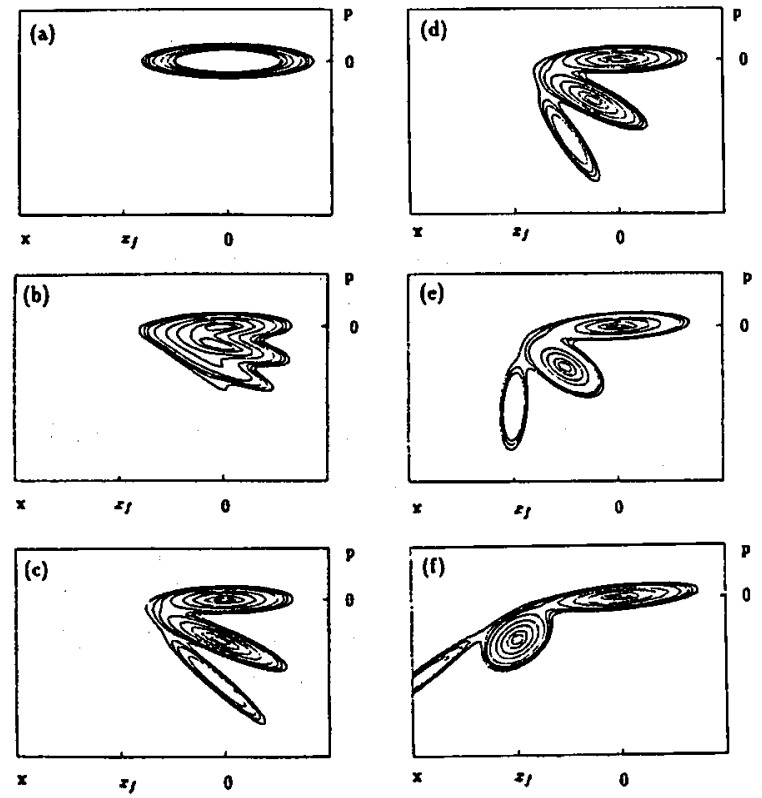

Fig. 4. Time evolution of the Wigner function of the atom in the cavity ((a)-(c)) and outside the light field ((d)-(f)). Here we display the contour lines of the three dominant peaks corresponding to the vacuum $n=0$ and the first two excited Fock states $n=1$ and $n=2$. The field is in a coherent state of average number of photons $\bar{n}=1$. In (a) we display the initial distribution, and in (b)-(c) we show the rotation and the splitting during the interaction with the light field. During the free evolution shown in (d)-(f) the individual cigars go through $x=x_{f}$ at different times corresponding to different focal distances. 
is weighted with the photon statistics. Figure $4 \mathrm{c}$ depicts the distribution of atoms at the exit of the cavity and serves as the initial distribution for the free evolution shown on the right column for various positions $z$ outside of the light field. Each cigar experiences the shearing effect discussed in Fig. 2. Moreover, different cigars move through the focal line $x=x_{f}$ at different times $t$, which corresponds to different positions $\mathcal{F}_{n}$.

\section{Distributions of atoms in space and in momentum}

So far we have analyzed the distribution of transverse position and momentum in its dependence on the coordinate $z$. In the present section we consider the spatial distribution $W(x, z)$ of atoms in the $x-z$ plane. We find it by integrating $P^{(\mathrm{W})}(x, p, z)$ given by Eq. (12) and Eq. (27) over $p$ and arrive at

$$
W(x, z)=\sum_{n=0}^{\infty}\left|w_{n}\right|^{2} W_{n}(x, z)
$$

where $W_{n}(x, z) \equiv \int \mathrm{d} p P_{n}^{(\mathrm{W})}(x, p, z)$ is the spatial distribution of atoms due to the interaction with the $n$-th Fock state. After evaluation of the Gaussian integrals we find

$$
\begin{aligned}
W_{n}(x, z) & =\frac{1}{\sqrt{\pi} D_{n}(z)} \\
& \times \exp \left\{-\frac{1}{D_{n}^{2}(z)}\left[x-x_{f}\left(1-\cos \varphi_{n}+\frac{\omega_{n}}{v_{z}} z \sin \varphi_{n}\right)\right]^{2}\right\}
\end{aligned}
$$

with

$$
\begin{gathered}
D_{n}(z)=\left[\left(\frac{\hbar}{d M \omega_{n}}\right)^{2}\left(\frac{\omega_{n}}{v_{z}} z \cos \varphi_{n}+\sin \varphi_{n}\right)^{2}\right. \\
\left.+d^{2}\left(\cos \varphi_{n}-\frac{\omega_{n}}{v_{z}} z \sin \varphi_{n}\right)^{2}\right]^{1 / 2} .
\end{gathered}
$$

In Fig. 5 we show the contour lines of the distribution $W(x, z)$ of atoms and a cut along the focal line $x=x_{f}$ for a coherent state of average number of photons $\bar{n}=1$. We note that the initial atomic beam splits up into a number of partial beams due to the deflection of atoms by the individual Fock states. Moreover, we find that each partial beam corresponding to the $n$-th Fock state focuses at the individual point $\left(x_{f}, \mathcal{F}_{n}\right)$. The cut through the distribution $W(x, z)$ along the focal line $x=x_{f}$ depicted in the right part of Fig. 5 shows the weight of each partial beam reflecting the photon statistics of the initial field state.

We obtain the focal length $\mathcal{F}_{n}$ from Eq. (30) as the position $z$ where $D_{n}(z)$ reaches the minimum. For $z>0$, that is outside of the light field this corresponds to $\cos \varphi_{n}-\left(\omega_{n} / v_{z}\right) z \sin \varphi_{n}=0$, which yields

$$
z_{f}=\mathcal{F}_{n}=\frac{v_{z}}{\omega_{n} \tan \varphi_{n}} \text {. }
$$

When the atom leaves the light field at $z=0$, the centre of the wave paciet is located at

$$
x_{n}=x_{f}\left(1-\cos \varphi_{n}\right) \text {. }
$$



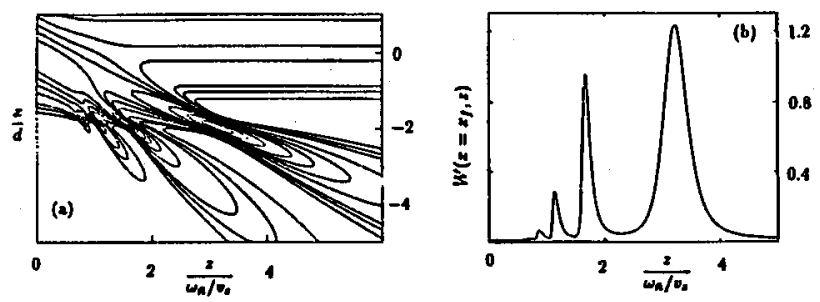

Fig. 5. Contour plot (a) of the probability $W(x, z)$ of finding an atom at the point with coordinates $x$ and $z$. The Gaussian atomic beam centered at $x=0$ leaves the cavity at $z=0$. The field is in a coherent state of average number of photons $\bar{n}=1$. The undeflected and unfocused partial wave associated with the cavity vacuum state represents the profile of the incident beam. The deflected partial waves associated with different photon states of the field focus along the line $x=x_{f}$. The intensity of atoms along this line is shown in (b). The parameters used here are: $\varphi_{\bar{n}}=0.3, \hbar / d^{2} M \omega_{\bar{n}}=20$, $x_{f}=-2$.

It passes through the focus $x=x_{f}$ and $z=\mathcal{F}_{n}$ and hence experiences a deflection by an angle

$$
\dot{\theta_{n}}=\arctan \left(\frac{x_{f}-x_{n}}{\mathcal{F}_{n}}\right)=\arctan \left(x_{f} \frac{\omega_{n}}{v_{z}} \sin \varphi_{n}\right) .
$$

The focal distance $\mathcal{F}_{n}$ given by Eq. (31) depends on the curvature $g_{2}$ of the potential $V_{n}$, since $\omega_{n}=\sqrt{n g_{2} / M}$ and $\varphi_{n}=\omega_{n} L / v_{z}$ depend on $g_{2}$. In contrast, the deflection angle $\theta_{n}$ depends on the slope $g_{1}$ as well as on the curvature $g_{2}$. IIowever in the limit of $\varphi_{n} \ll 1$, the angle $\theta_{n}$ becomes independent of $g_{2}$, that is

$$
\theta_{n}=\arctan \left(\frac{n}{N}\right),
$$

where

$$
\frac{1}{N}=\frac{g_{1} L}{M v_{z}^{2}}
$$

is the deflection due to the first Fock state. Moreover, in this limit the focal length reads

$$
\mathcal{F}_{n} \approx \frac{1}{n} \mathcal{F}_{1}
$$

where

$$
\mathcal{F}_{1}=\frac{M v_{z}^{2}}{g_{2} L}
$$

is the focal length of the Fock state $\mid n=1$ ).

We note that we can obtain the photon statistics making use of the strong correlation between the field state and the momentum distribution $\widetilde{W}(p)$ of the atoms after they have left the field. We find this distribution by integration of $P^{(\mathrm{W})}(x, p, t)$ over $x$ which yields

$$
\widetilde{W}(p)=\sum_{n=0}^{\infty}\left|w_{n}\right|^{2} \widetilde{W}_{n}(p) \text {. }
$$


Here

$$
\widetilde{W}_{n}(p)=\frac{1}{\sqrt{\pi} \widetilde{D}_{n}} \exp \left[-\left(\frac{d}{\hbar \widetilde{D}_{n}}\right)^{2}\left(p-M \omega_{n} x_{f} \sin \varphi_{n}\right)^{2}\right]
$$

with

$$
\widetilde{D}_{n}=\left[\cos ^{2} \varphi_{n}+\left(\frac{d^{2} M \omega_{n}}{\hbar} \sin \varphi_{n}\right)^{2}\right]^{1 / 2}
$$

is the momentum distribution of the atoms due to the interaction with the $n$-th Fock state. An atom which has entered the light field in the $n$-th Fock state with the average momentum $\langle p\rangle=0$ leaves the cavity with the average momentum

$$
p_{n}=M \omega_{n} x_{f} \sin \varphi_{n},
$$

as indicated by the maximum of the momentum distribution $\widetilde{W}(p)$, Eq. (39). The motion in the free field region conserves the momentum and hence the distribution is independent of the position $z$. It is this momentum transfer which gives rise to the deflection angle $\theta_{n}$, Eq. (33), via the relation $\theta_{n}=\arctan \left(p_{n} / M v_{z}\right)$.

When the difference $\Delta p_{n}=p_{n+1}-p_{n}$ in the transferred momentum due to two neighboring Fock states is larger than the momentum uncertainty given by the width $\widetilde{D}_{n}$ of the two corresponding Gaussians, we can resolve the contribution of each individual Fock state. Since each Gaussian is weighted with the photon statistics, the momentum distribution in this case is a completc rcadout of the photon statistics as shown in Fig. 6.
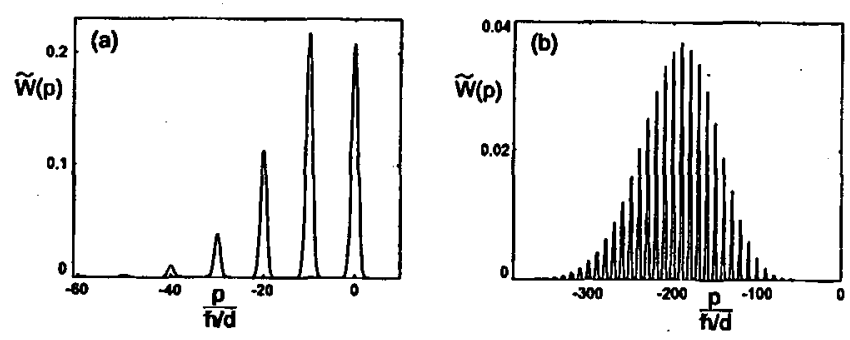

Fig. 6. Momentum distribution of atoms scattered by a quantized electromagnetic field of a coherent state of a verage number of photons $\bar{n}=1$ (a) and $\bar{n}=20$ (b). We can identify the contributions of the individual Fock states comprising the coherent state. The envelope of the momentum distribution gives the Poisson photon statistics. Here we have chosen: for (a) $\varphi_{\bar{n}}=1 / \sqrt{10}, \hbar / d^{2} M \omega_{\bar{n}}=\sqrt{10}$, for (b) $\varphi_{\bar{n}}=\sqrt{2}, \hbar / d^{2} M \omega_{\bar{n}}=1 / \sqrt{2}$ and in both cases $x_{f}=-100$.

We note that in deriving the above results we have expanded the coupling constant $g(x)$ around the centre of the wave packet. Hence this solution is only valid provided the wave packet has not moved considerably due to the interaction with the light field, that is it has not moved into a regime in which the displaced harmonic oscillator potential is not a good approximation to the potential $V_{n}$. 
Hence the approximation is valid if the cubic term of the expansion is negligible, that is

$$
\left.\frac{1}{3 !} g_{3} x^{3} \equiv \frac{1}{3 !} \frac{\mathrm{d}^{3} g}{\mathrm{~d} x^{3}}\right|_{x=0} x^{3} \ll \frac{1}{2 !} g_{2} x^{2} .
$$

This condition puts the constraint

$$
|x| \ll 3\left|\frac{g_{2}}{g_{3}}\right|
$$

on the $x$-values in which the atom is allowed to move in order to uphold the quadratic approximation Eq. (16). Hence the displacement, Eq. (32), and the width $D_{n}(z=0)$, Eq. $(30)$, of the exiting atomic wave packet have to be within this region.

We conclude this section by comparing the spatial distribution $W(x, z)$ of atoms, Eqs. (28) $-(30)$, in the $x-z$ plane to the distribution

$$
W_{n}^{(\mathrm{RN})}(x, z)=\frac{1}{\sqrt{\pi} D_{n}^{(\mathrm{RN})}(z)} \exp \left[-\left(\frac{n z+N x}{N D_{n}^{(\mathrm{RN})}(z)}\right)^{2}\right]
$$

with

$$
D_{n}^{(\mathrm{RN})}(z)=\left[\left(\frac{\hbar}{d M v_{z}} z\right)^{2}+d^{2}\left(1+\frac{n}{N} \frac{z}{x_{f}}\right)^{2}\right]^{1 / 2}
$$

obtained [9] in the Raman-Nath approximation. We note that Eq. (29) reduces to Eq. (44) in the limit of small rotation angles $\varphi_{n}=\omega_{n} t_{L} \ll 1$, such that $\cos \varphi_{n} \approx 1$ and $\sin \varphi_{n} \approx \varphi_{n}$. However, the width $D_{n}$, Eq. (30), reduces to

$$
D_{n}(z) \approx\left\{\left[\frac{\hbar}{d M v_{z}}(z+L)\right]^{2}+d^{2}\left(1+\frac{n z}{N x_{f}}\right)^{2}\right\}^{1 / 2}
$$

and hence contains an additional term $\hbar L / d M v_{z}$ which reflects the spreading of the wave packet during the motion of the atom in the harmonic oscillator potential. The Raman-Nath approximation neglects the kinetic part of the Hamiltonian compared to the electromagnetic interaction energy and hence cannot account for this term.

\section{Focal size and focal length}

In the preceding sections we have discussed the complete phase space dynamics of the atomic motion in the quantized light field. In the present section we illustrate this approach in simple geometrical terms. In particular we rederive the expression Eq. (31) for the focal distance $\mathcal{F}_{n}$ of the $n$-th Fock state quantum lens starting from the pictorial sketch, and calculate the size of the focal spot.

Figure 7 explains the essential points of the process of focusing. On the right hand side we summarize the set-up whereas on the left hand side we show the evolution of the atomic wave packet in phase space. The atomic wave of transverse width $\Delta x$, depicted here by a fat line, enters the light field (shaded area) at $z=-L$ and leaves it slightly displaced and compressed at $z=0$. Due 

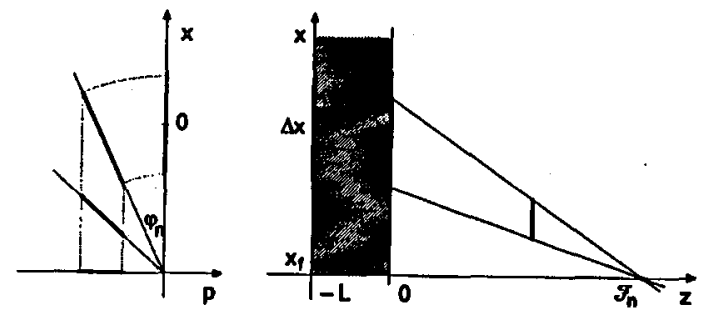

Fig. 7. Quantum lens due to the $n$-th Fock state (right) and evolution of the atomic beam in phase space (left). Here we only consider an interval $\Delta x$ of the $x$-axis. Due to the interaction with the standing light field this interval is rotated by an angle $\varphi_{n}$. In the limit of small angles $\varphi_{n} \ll 1$ the displacement in $x$-direction is small compared to $\Delta x$. During the free evolution the considered points in phase space remain on a straight line and cross the focal line $x=x_{f}$ at the same time $t_{f}$. As a result the width $\Delta x$ of the wave packet decreases until it vanishes at $x=x_{f}$.

to the interaction with the light in the $n$-th Fock state different parts of the wave packet gain different transverse momenta. As a result of the free evolution outside of the cavity the beam focuses at the point $z=\mathcal{F}_{n}$. This process is most easily understood from the dynamics in phase space. For the sake of simplicity we take the initial phase space distribution to be a line distribution of length $\Delta x$ centered at $x=0$ with vanishing momentum. Since the potential formed by the $n$-th Fock state is harmonic, the fat line representing the initial distribution rotates in phase space by an angle $\varphi_{n}$ around $\left(x=x_{f}, p=0\right)$. This rotation in phase space is the origin of the slight shift and the compression of the wave packet. Moreover, different parts of the wave packet acquire different momenta which are proportional to their coordinates. Outside of the cavity the momentum of each part is conserved. As a result all points of the rotated line distribution pass the point $x=x_{f}$ at the same time $t_{f}$ as shown by the fat line along the momentum axis. Since time corresponds to the $z$-coordinate via the relation $z=v_{z} t-L$, the focal length $\mathcal{F}_{n}^{\prime}$ of the $n$-th Fock state reads $\mathcal{F}_{n}=v_{z} t_{f}-L$.

Now we get the focal distance $\mathcal{F}_{n}$, Eq. (31), using this picture. For this purpose we consider the time evolution of phase space points with vanishing initial momentum, that is $p(t=0)=0$ as shown on the left hand side of Fig. 7 by the fat line centered at $x=0$. At a later time $t \leq t_{L}$ these points have moved to the new positions

$$
\begin{aligned}
& x(t)=\left(x_{0}-x_{f}\right) \cos \left(\omega_{n} t\right)+x_{f}, \\
& p(t)=-M \omega_{n}\left(x_{0}-x_{f}\right) \sin \left(\omega_{n} t\right),
\end{aligned}
$$

and now lie on the line

$$
p(x)=-M \omega_{n} \tan \left(\omega_{n} t\right)\left(x-x_{f}\right)
$$

in phase space which goes through the point $x=x_{f}$ at the angle $\varphi_{n}=\omega_{n} t$ with respect to the $x$-axis. After the atom has left the light field it moves freely and conserves its momentum

$$
p_{L} \equiv p\left(t \geq t_{L}\right)=-M \omega_{n} \tan \left(\varphi_{n}\right)\left(x_{L}-x_{f}\right),
$$


where $x_{L} \equiv x\left(t_{L}\right)$, and the position $x$ evolves according to

$$
x(t)=\frac{p_{L}}{M}\left(t-t_{L}\right)+x_{L}=\left[1-\omega_{n} \tan \left(\varphi_{n}\right)\left(t-t_{L}\right)\right]\left(x_{L}-x_{f}\right)+x_{f} .
$$

Therefore all points on the line Eq. (47) reach the focal spot $x=x_{f}$ at the same time

$$
t_{f}=\frac{1}{\omega_{n} \tan \left(\varphi_{n}\right)}+t_{L}
$$

The corresponding distance in $z$-direction yields the desired focal length

$$
\mathcal{F}_{n} \equiv v_{z} t_{f}-L=\frac{v_{z}}{\omega_{n} \tan \left(\varphi_{n}\right)} .
$$

This expression is identical to the one, Eq. (31), obtained from the spatial distribution $W(x, z)$ of the atoms in Sec. 4 .

So far we have considered the extreme of geometrical optics, that is a line distribution in phase space which is infinitely narrow in momentum. But what happens to the focus in the framework of wave optics, that is when we admit a finite uncertainty $\Delta p$ in momentum? It results in a spreading of the focal point, that is a width of the focus. Indeed, we estimate $\Delta p$ for a wave packet with the width $d$ in position space via the Heisenberg-relation

$$
\Delta p \approx \frac{\hbar}{d}
$$

and consider the time evolution of the phase space point $(x=0, p=\Delta p)$. The time $t_{f}^{\prime}$ at which this point crosses the line $x=x_{f}$ provides via the relation $z_{n}^{\prime}=v_{z} t_{f}^{\prime}-L$ the width $\delta \mathcal{F}_{n}=z_{n}^{\prime}-\mathcal{F}_{n}$. At time $t_{L}$ the point $(x=0, p=\Delta p)$ has moved to

$$
x_{L}^{\prime}=-x_{f} \cos \varphi_{n}+\frac{\hbar}{M \omega_{n} d} \sin \varphi_{n}+x_{f}
$$

and

$$
p_{L}^{\prime}=x_{f} M \omega_{n} \sin \varphi_{n}+\frac{\hbar}{d} \cos \varphi_{n} .
$$

We now find the time $t_{f}^{\prime}$, at which this point passes the focal line $x=x_{f}$. Since the momentum $p_{L}^{\prime}$ is conserved during the free evolution we find

$$
\frac{p_{L}^{\prime}}{M}\left(t_{f}^{\prime}-t_{L}\right)+x_{L}^{\prime}=x_{f}
$$

and hence

$$
t_{f}^{\prime}=t_{L}+\frac{M}{p_{L}^{\prime}}\left(x_{f}-x_{L}^{\prime}\right)
$$

This time translates into

$$
z_{n}^{\prime}=v_{z} t_{f}^{\prime}-L=\frac{M v_{z}}{p_{L}}\left(x_{f}-x_{L}\right)
$$

and the width $\delta \mathcal{F}_{n}$ of the $n$-th focus reads

$$
\delta \mathcal{F}_{n}=z_{n}^{\prime}-\mathcal{F}_{n}=\mathcal{F}_{n}\left[\frac{d x_{f}-\Delta^{2} \tan \varphi_{n}}{d x_{f}+\Delta^{2} \cot \varphi_{n}}-1\right] .
$$

where $\Delta=\sqrt{\hbar / M \omega_{n}}$ denotes the spread of the atom in the ground state of the harmonic potential Eq. (17). 
We conclude this section by noting that in the limit of small angles $\varphi_{n} \ll 1$ Eq. (58) reduces to

$$
\delta \mathcal{F}_{n}=\mathcal{F}_{n}^{\prime}-\mathcal{F}_{n} \approx \frac{M \hbar v_{z}^{3}}{d g_{1} g_{2} L^{2}} \frac{1}{n^{2}}
$$

a result derived in Ref. [9].

\section{Summary}

In the present paper we have considered the atomic motion in a quantized field. Here we have concentrated on the atom by tracing over the field variables. We have shown that the Wigner functions of the atom corresponding to the motion in the potentials due to the individual Fock states separate in phase space. This separation manifests itself either in the momentum distribution as a deflection in different angles or in the spatial distribution as focusing in different points.

\section{References}

[1] M. Hillery, R.F. O'Connell, M.O. Scully, E.P. Wigner, Phys. Rep. 106, 122 (1984).

[2] For a review see C. Cohen-Tannoudji, in: Fundamental Systems in Quantum Optics, Eds. J. Dalibard, J.M. Raimond, J. Zinn-Justin, North-Holland, Amsterdam 1992, p. 1.

[3] For a review of the field of atom optics, see the special issue Appl. Phys. $B$ 54 (April 1992), Optics and Interferometry with Atoms, Eds. J. Mlynek, V. Balykin, P. Meystre; S. Stenholm, in Lectures at the CXVIII International School of Physics Laser Manipulation of Atoms and Ions, Eds. E. Arimondo, W.D. Phillips, F. Strumia, Proc. International School of Physics "Enrico Fermi", Course CXVIII, Varenna, 1991. The experimental situation is summarized by M. Sigel, C.S. Adams, J. Mlynek, in: Frontiers in Laser Spectroscopy, Eds. T.W. Hänsch, M. Inguscio, Proc. International School of Physics "Enrico Fermi", Course CXX, Varenna, 1992.

[4] A.P. Kasantsev, G.I. Surdutovich, V.P. Yakovlev, Mechanical Aclion of Light on Atoms, World Scientific, Singapore 1990.

[5] For a discussion of the Wigner function for a particle with internal degrees of freedom, see for example V.M. Akulin, N.V. Karlov, Intense Resonant Interactions in Quantum Electronics, Springer, Berlin 1992 or S. Stenholm, Phys. Rev. A 47, 2523 (1993).

[6] P. Meystre, E. Schumacher, S. Stenholm, Opt. Commun. 73, 443 (1989).

[7] V.M. Akulin, Fam Le Kien, W.P. Schleich, Phys. Rev: A 44, R1462 (1991).

[8] A.M. Herkommer, V.M. Akulin, W.P. Schleich, Phys. Rev. Lett. 69, 3298 (1992).

[9] I.Sh. Averbukh, V.M. Akulin, W.P. Schleich, Phys. Rev. Lelt. 72, 437 (1994).

[10] M. Brune, S. Haroche, V. Lefevre, J.M. Raimond, N. Zagury, Phys. Rev. Lett. 65, 976 (1990).

[11] M.J. Holland, D.F. Walls, P. Zoller, Phys. Rev. Lett. 67, 1716 (1991).

[12] P. Storey, M. Collett, D. Walls, Phys. Rev. Lett. 68, 472 (1992).

[13] E. Kamke, Differentialgleichungen: Lösungsmethoden und Lösungen, Band II: Partielle Differentialgleichungen, 6. Auflage, B.G. Teubner, Stuttgart 1979. 